\title{
Eventos prejudiciais à reputação corporativa: um estudo exploratório utilizando a técnica de vinhetas
}

\author{
Corporate reputation damage events: an exploratory study using the \\ vignettes technique
}

\author{
Dr. José Carlos Hoelz, Universidade Nove de Julho, São Paulo, Brasil. \\ jchoelz@gmail.com | http://orcid.org/ 0000-0002-4079-5907 \\ Av. Professor Luiz Ignácio Anhaia Mello, 1363 - Vila Prudente, São Paulo - SP, 03155-000, Brasil \\ Dr. Walter Bataglia, Universidade Presbiteriana Mackenzie, São Paulo, Brasil. \\ batagliaw@gmail.com | http://orcid.org/ 0000-0003-4233-9988 \\ Av. Mackenzie, 905 - Tamboré, Barueri SP - Brasil
}

\begin{abstract}
Resumo
Este artigo tem como objetivo explorar, por meio de uma investigação qualitativa, a percepção de gestores de alianças estratégicas sobre como eventos prejudiciais à reputação de parceiros de negócios afetam as parcerias. Além disso, este artigo se propõe a discutir a utilização de vinhetas como uma técnica a ser utilizada em um estudo exploratório. Para atingir este objetivo, foi operacionalizado um estudo que teve como contexto o setor farmacêutico, no qual foram realizadas entrevistas em quatro empresas brasileiras. Na coleta de dados foram utilizadas vinhetas. A técnica de vinhetas é ideal para ser utilizada em situações em que é preciso compreender o julgamento do participante sobre uma determinada situação-estímulo. Neste sentido, nas entrevistas, buscou-se explorar a percepção de gestores de alianças estratégicas com relação a eventos prejudiciais à reputação corporativa do parceiro nas alianças. As transcrições literais, resultantes das entrevistas, foram analisadas com o uso do aplicativo NVivo, nas etapas de preparação dos dados, codificação, análise das respostas dos entrevistados e elaboração do relatório para apresentação dos resultados. Como principal resultado obtido destaca-se o papel da repercussão de um evento prejudicial à reputação nos meios comunicação como um importante determinante para a tomada de decisão dos gestores de alianças estratégicas. Este estudo também foi bem sucedido em demonstrar que o uso de vinhetas de maneira qualitativa é uma técnica adequada para ser utilizada em estudos exploratórios.
\end{abstract}




\begin{abstract}
In this article we aim to explore, through a qualitative research, the perception of strategic alliance managers about how corporate reputation damage events affect these alliances. Additionally, we aimed to discuss the use of vignettes as a technique to be used in exploratory studies. To achieve this goal, we conducted a study in the pharmaceutical sector context, where interviews were conducted with four managers from Brazilian companies. In the data collection, vignettes were used. The technique of vignettes is ideal to be used in situations where it is necessary to understand the judgment of the participant in a given stimulus situation. In this sense, in these interviews, we sought to explore the perception of strategic alliance managers regarding corporate damage events in strategic alliances. The literal transcripts resulting from the interviews were analyzed with NVivo software in the stages of data preparation, coding, analysis of interviewees' responses and preparation of the report for presentation of the results. 'About the main result obtained, stands out the role of repercussion in media as an important and determinant factor to decision making process of alliance managers. This study was also successful in demonstrating that the use of vignettes in a qualitative way is a suitable technique to be used in exploratory studies.
\end{abstract}

Palabras clave: eventos prejudiciais à reputação; vinhetas; investigação qualitativa; pesquisa exploratória.

Keywords: corporate reputation damage events; vignettes; qualitative research; strategic alliance

\title{
1. INTRODUÇÃO
}

Dentre os vários elementos que podem ser considerados nas alianças estratégicas, é importante destacar o papel da reputação corporativa. A reputação corporativa é um elemento suplementar ao processo de governança das alianças estratégicas, que pode ser interpretado como uma forma de reduzir a incerteza relacionada ao comportamento de cooperação nesses tipos de relações interorganizacionais (Hirsch \& Meyer, 2010).

De acordo com o Reputation Leaders Study (2016), um estudo realizado com 150 executivos de comunicação corporativa em 20 países, $59 \%$ dos entrevistados dizem que a reputação corporativa tem prioridade alta para as empresas. Também, $45 \%$ dos executivos respondentes indicam que a reputação corporativa é um item proeminente da agenda da alta administração e $77 \%$ dos entrevistados consideram os resultados financeiros da empresa entre as métricas que refletem sua reputação corporativa.

Os dados apresentados corroboram a ideia de que a construção de uma reputação corporativa positiva é elemento essencial para a estratégia das organizações. Entretanto, a reputação corporativa de uma organização pode ser afetada de maneira negativa por eventos prejudiciais, ou seja, problemas que causam um retrocesso nas percepções das partes interessadas (Rhee \& Kim, 2012) e resultam em prejuízos. 
Desta maneira, este estudo tem como objetivo explorar, por meio de uma investigação qualitativa, a percepção de gestores de alianças estratégicas sobre como eventos prejudiciais à reputação de parceiros de negócios afetam as parcerias. Para alcançar este objetivo, utilizou-se a técnica de vinhetas para a condução de entrevistas com quatro executivos de alianças estratégicas de quatro empresas do setor farmacêutico brasileiro. Após, foi realizada a análise de categorias (Flores, 1994) das transcrições resultantes, utilizando-se o aplicativo NVivo, versão 11.

Como resultados principais deste artigo podem-se destacar as contribuições para o aprofundamento da compreensão sobre como as dimensões dos eventos prejudiciais à reputação afetam as percepções dos gestores de alianças estratégicas, em especial, sobre o efeito da repercussão destes eventos no processo de tomada de decisão destas alianças. Também, contribuiu-se para o entendimento das implicações teóricas e metodológicas do uso da técnica de vinhetas no âmbito da investigação qualitativa na pesquisa em ciências sociais aplicadas.

Após esta introdução, o texto foi organizado em seis seções: a primeira se ocupa em discutir o conceito de eventos prejudicais à reputação corporativa e sobre os diversos aspectos acerca do uso de vinhetas em uma investigação qualitativa. A segunda, descreve o contexto do estudo, o setor farmacêutico brasileiro. A terceira, descreve a metodologia empregada no estudo. Na quarta seção, é feita a análise das categorias. As discussões sobre os resultados são feitas na quinta seção. Para finalizar, na sexta seção, ao concluir o estudo, são feitas algumas considerações sobre as contribuições teóricas e a respeito das possibilidades e limites do uso da técnica de vinhetas em pesquisa.

\section{REVISÃO TEÓRICA}

\subsection{Eventos Prejudiciais à Reputação Corporativa}

A reputação corporativa descreve a percepção agregada de julgamentos múltiplos das várias partes interessadas a respeito do desempenho das organizações (Fombrun, 1996; Fombrun, Gardberg, \& Sever, 2000). Esta percepção é construída por meio das experiências que as partes interessadas têm ao interagirem com a organização e com os seus produtos e serviços. Quando a experiência é positiva, a percepção gera a satisfação das partes interessadas.

Estudos mostram que uma forte reputação positiva é um elemento intangível (Wagner, Coley, \& Lindemann, 2011) da estratégia. Tal elemento promove a vantagem competitiva sustentável (Barney, 1991), contribui para o desempenho econômico da firma (Fombrun, 1996; Fombrun \& Shanley, 1990; Roberts \& Dowling, 2002) e torna significativamente mais fácil estabelecer novos relacionamentos (Feldman, Bahamonde, \& Bellido, 2014; Sydow, 1998), como, por exemplo, as alianças estratégicas (Dollinger, Golden, \& Saxton, 1997; Hirsch \& Meyer, 2010).

Um contraponto ao efeito positivo da reputação corporativa é a implicação negativa dos eventos prejudiciais à reputação das organizações. Apesar de ser fundamental para a competitividade e rentabilidade da empresa, a reputação corporativa é frágil e, às vezes, pode ser facilmente 
danificada por eventos que afetem negativamente as percepções das partes interessadas (Hutt, 2016). Assim, um evento prejudicial à reputação pode ser definido como um problema pelo qual uma organização enfrenta um retrocesso nas percepções das partes interessadas (Rhee \& Kim, 2012). Segundo Rhee e Kim (2012), os eventos prejudiciais à reputação têm três dimensões: o tipo do evento, a atribuição do evento e a raridade do evento. Estas dimensões estão descritas com mais detalhes nas linhas abaixo e podem ser vistas na Tabela 1.

Tabela 1. Dimensões dos eventos prejudicais à reputação

\begin{tabular}{|l|l|}
\hline Tipo do evento & $\begin{array}{l}\text { Crise organizacional, ameaças e eventos de alto impacto que podem prejudicar } \\
\text { a reputação corporativa. }\end{array}$ \\
\hline $\begin{array}{l}\text { Atribuição de } \\
\text { responsabilidade }\end{array}$ & $\begin{array}{l}\text { A maneira pela qual o evento é percebido pelas partes interessadas como uma } \\
\text { falha atribuída à organização focal. }\end{array}$ \\
\hline $\begin{array}{l}\text { Raridade do } \\
\text { evento }\end{array}$ & Os eventos prejudiciais à reputação geralmente são incomuns. \\
\hline
\end{tabular}

Fonte: elaborada pelo autor baseado em Rhee \& Kim (2012).

De acordo com Rhee e Kim (2012), os estudos que tipificam os eventos prejudiciais à reputação concentram-se basicamente na crise organizacional. A crise pode ser descrita como um evento imprevisível de alto impacto, com potencial para interromper as operações de uma organização e ameaçar ou prejudicar a sua reputação corporativa, causando danos financeiros e à reputação da organização (Coombs \& Holladay, 2002). Uma crise tem potencial para ameaçar a reputação de uma organização porque dá às pessoas motivos para pensarem mal desta organização (Coombs \& Holladay, 2002; Timothy \& Coombs, 2007). Em seu estudo sobre a Teoria Situacional de Comunicação de Crise, do inglês Situational Communication Crise Theory (SCCT), Timoty e Coombs (2007) indicam uma lista de 13 tipos de crise, que têm o potencial para prejudicar a reputação de uma organização. Esta tipificação está descrita na Tabela 2. 
Tabela 2: Tipos de crises com potencial para afetar a reputação corporativa

\begin{tabular}{|c|c|}
\hline Rumor & $\begin{array}{l}\text { Circulação de informações falsas destinadas a prejudicar } \\
\text { uma organização. }\end{array}$ \\
\hline Desastre Natural & $\begin{array}{l}\text { Um evento de ocorrência natural que casa danos à uma } \\
\text { organização. }\end{array}$ \\
\hline Malevolência/Adulteração de produtos & $\begin{array}{l}\text { Dano causado por um agente externo contra uma } \\
\text { organização. }\end{array}$ \\
\hline Violência no local de trabalho & $\begin{array}{l}\text { Um ataque realizado por um empregado ou ex-empregado } \\
\text { contra funcionários atuais no local de trabalho. }\end{array}$ \\
\hline Desafio & $\begin{array}{l}\text { Confronto com partes interessadas descontentes que } \\
\text { afirmam que a organização está operando de forma } \\
\text { inadequada. }\end{array}$ \\
\hline $\begin{array}{l}\text { Acidente devido a mau funcionamento } \\
\text { técnico }\end{array}$ & $\begin{array}{l}\text { Um acidente industrial causado por falhas na tecnologia ou } \\
\text { nos equipamentos. }\end{array}$ \\
\hline $\begin{array}{l}\text { Substituição do produto devido a mau } \\
\text { funcionamento técnico }\end{array}$ & $\begin{array}{l}\text { Substituição de um produto devido à falha na tecnologia ou } \\
\text { nos equipamentos. }\end{array}$ \\
\hline Megadano & $\begin{array}{l}\text { Um acidente devido à avaria técnica que produz danos } \\
\text { ambientais significativos. }\end{array}$ \\
\hline Acidente devido a falha humana & Acidente industrial causado por erro humano. \\
\hline $\begin{array}{l}\text { Substituição de produto devido à falha } \\
\text { humana }\end{array}$ & Substituição de produto causado por erro humano. \\
\hline $\begin{array}{l}\text { Delitos organizacionais sem lesões } \\
\text { (para partes interessadas externas) }\end{array}$ & $\begin{array}{l}\text { A gestão conscientemente engana as partes interessadas, } \\
\text { mas sem causar feridos. }\end{array}$ \\
\hline $\begin{array}{l}\text { Delito organizacional causado por má } \\
\text { conduta gerencial }\end{array}$ & $\begin{array}{l}\text { A administração conscientemente viola leis ou regulamen- } \\
\text { tos. }\end{array}$ \\
\hline $\begin{array}{l}\text { Delitos organizacionais com lesões } \\
\text { (para partes interessadas externas) }\end{array}$ & $\begin{array}{l}\text { A administração conscientemente engana as partes } \\
\text { interessadas causando ferimentos. }\end{array}$ \\
\hline
\end{tabular}

Fonte: elaborada pelo autor baseado em Coombs \& Holladay, 2002 e Timothy \& Coombs, 2007. 
Com relação à atribuição dos eventos prejudiciais à reputação, estes variam na medida em que são atribuídos a uma organização focal. Se um evento prejudicial à reputação for percebido como culpa da empresa, haverá uma percepção mais forte de que esta deve ser responsável pelo evento, o que resulta em maiores danos à reputação da organização (Rhee \& Kim, 2012).

A respeito da raridade dos eventos prejudiciais à reputação, Rhee e Kim (2012) argumentam que tais eventos não são comuns, mas que, apesar disso, as organizações devem concentrar o seu esforço na resolução deste tipo de problema, devido às lições úteis que podem ser aprendidas com eles. As características únicas de cada tipo de evento guardam lições valiosas para a prevenção de danos à reputação das organizações.

Os eventos prejudiciais à reputação podem danificar a reputação corporativa de uma organização, afetando negativamente as percepções das partes interessadas sobre a capacidade da organização para cumprir com êxito as suas obrigações. Assim, os eventos prejudiciais à reputação têm o potencial de afetar as percepções das partes interessadas, uma vez que estes eventos dão visibilidade externa a respeito de falhas (Rhee \& Valdez, 2009). Os eventos prejudiciais tendem a afetar as percepções sobre as perspectivas de futuro de uma organização e a criar maior incerteza ambiental. Isso se deve ao fato de que, muitas vezes, esses eventos prejudicam a credibilidade das organizações, particularmente quando existem fraudes envolvidas. Eventos prejudiciais à reputação acabam por resultar em erosão da confiança percebida, acarretando perda de credibilidade das empresas (Gertsen, Van Riel, \& Berens, 2006).

Por fim, uma vez que os eventos prejudiciais à reputação afetam negativamente a reputação corporativa de uma organização, trazem, por consequência, repercussões para o desempenho das alianças estratégicas. Considerando a ideia de que uma boa reputação pode ser prejudicada, é importante para as organizações determinarem qual o nível de impacto que os eventos prejudiciais à reputação têm sobre o seu desempenho. Existem estudos que demonstram que os eventos prejudiciais à reputação produzem efeitos significativos sobre o desempenho financeiro, dependendo do tipo de evento. Estes impactos são mais significativos notadamente no caso de fraudes (Gatzert, 2015). Os eventos prejudiciais à reputação, quando ocorrem, podem trazer consequências financeiras, que vão além do prejuízo real para a reputação, como, por exemplo, a redução dos lucros, clientes ou a perda de parceiros de negócios (Gatzert, Schmit, \& Kolb, 2016).

\subsection{O Uso de Vinhetas em Estudos Qualitativos}

As vinhetas são tidas como uma técnica de coleta de dados que utiliza cenários ou histórias curtas em forma escrita ou pictórica, sobre as quais os participantes podem comentar e cuja principal característica é explorar os sistemas de crenças subjetivas desses participantes (Renold, 2002). Ainda, na literatura, é possível discernir os seguintes aspectos associadas à elaboração de vinhetas: sobre a maneira como são construídas; com relação ao seu conteúdo; sobre a sua forma de aplicação; e com relação a sua finalidade. 
Em contraste com a aplicação de vinhetas em pesquisas de natureza quantitativa, que integradas ao uso de questionários de larga escala são limitadas a descrições gerais e pré-definidas, em detrimento de descrições mais específicas das atitudes envolvidas em uma dada situação, o uso de vinhetas qualitativas permite aos participantes definirem uma dada situação em seus próprios termos (Barter \& Renold, 1999, 2000; Renold, 2002).

Essa característica enfatiza a capacidade dessa técnica em explorar sistemas de crenças subjetivas dos participantes (Crafter, De Abreu, Cline, \& O’Dell, 2010; Hughes, 1998)2001. Por esta razão, as vinhetas qualitativas são utilizadas em estudos relacionados à pesquisa de normas culturais derivadas das atitudes dos entrevistados, em estudos sobre crenças, sobre códigos de enquadramento moral e éticos dos entrevistados, especialmente quando se usa mais de uma vinheta e a variação da história com relação à etnia e gênero (Barter \& Renold, 1999; Finch \& Mason, 1991; Renold, 2002). Ainda, em uma perspectiva qualitativa, as vinhetas podem ser utilizadas como instrumento de pesquisa longitudinal eficaz, destacando as mudanças nos marcos interpretativos ao longo do tempo, permitindo assim a melhor compreensão de tais mudanças (Jenkins, 2006).

É importante ressaltar que as vinhetas podem ser usadas de várias maneiras diferentes em pesquisas de natureza qualitativa, e a escolha sobre qual é a melhor forma de utilização envolve a opção teórica e metodológica de cada pesquisador. Portanto, esta escolha requer uma cuidadosa consideração sobre a melhor abordagem do uso da técnica (Barter \& Renold, 1999, 2000; Renold, 2002).

As vinhetas têm sido usadas de maneira qualitativa na área de administração de empresas em estudos sobre o conhecimento organizacional (Mariano, 2018), a respeito de como os atores corporativos de elite concebem e se preparam para o futuro (Berg Johansen \& De Cock, 2018) e sobre o gerenciamento de mudanças (Ashta, Stokes, \& Hughes, 2018).

\subsubsection{A elaboração de vinhetas}

Sobre a maneira como são construídas, as vinhetas podem ser elaboradas por meio de descrições focadas, ou de uma série de eventos, e são tomadas para serem representações típicas ou emblemáticas de um caso/situação (Miles \& Huberman, 1994). Ainda, elaboram-se as vinhetas por meio de: a) esboços compactos que podem ser usados para introduzir personagens, prenunciar eventos e análises futuras, destacar descobertas particulares, ou resumir um determinado tema ou assunto (Spalding \& Phillips, 2007); b) vídeos ou imagens de pessoas, situações e estruturas que podem fazer referência a pontos importantes do estudo de percepções, crenças e atitudes (Eskelinen \& Caswell, 2006; Hughes \& Huby, 2001; Nicholas Jenkins, Bloor, Fischer, Berney, \& Neale, 2010); c) um cenário que se desenvolve em uma série de estágios, nos quais os participantes são convidados a comentar sobre cada evolução da progressão da história (Hughes, 1998); e d) sob a perspectiva de um estudo interativo, no qual as escolhas dos participantes afetam o desenrolar da história (Jenkins, 2006). 
Com relação ao conteúdo, as vinhetas devem oferecer referências concretas, precisas e da forma mais detalhada possível, para aquilo que se imagina serem representações dos fatores de maior importância para o processo decisório dos participantes (Alexander \& Becker, 1978). Estas referências podem representar uma dada situação social que poderia ser real (Alexander \& Becker, 1978; Lieberman, 1987; Wright, Heathcote, \& Wibberley, 2014), ou sobre personagens hipotéticos em situações específicas (Finch \& Mason, 1991).

Com respeito à forma de aplicação, as vinhetas podem ser empregadas isoladamente ou de maneira integrada, com outras técnicas de pesquisa (como, por exemplo, a observação ou a entrevista), o que aumenta as possibilidades de geração dos dados. As vinhetas podem ser aplicadas no início da entrevista para facilitar a discussão, ou como um exercício de aquecimento para desenvolver o relacionamento entre os participantes e introduzir um tópico particular, sendo uma forma especialmente útil para abordar temas sensíveis ou desconfortáveis como, por exemplo, a "normalidade" (Barter \& Renold, 1999; Renold, 2002).

Sobre a sua finalidade, as pesquisas que utilizam a técnica de vinhetas devem buscar identificar o julgamento do respondente sobre o que seria próprio fazer em uma dada situação social de interesse (Barter \& Renold, 2000). Assim, os participantes serão convidados a fazer enunciados sobre o conjunto de circunstâncias que envolvem uma situação estudada (Finch \& Mason, 1991; Gourlay et al., 2014). As vinhetas são uma forma eficiente de suscitar uma discussão, identificar perspectivas diferentes, estimular a solução de problemas, promover a tomada de decisões e iniciar um processo de reflexão (Tettegah, Bailey, \& Taylor, 2007).

Finalmente, a literatura indica um conjunto de sete fatores que devem ser considerados no desenvolvimento de uma vinheta: (1) cada situação-estímulo oferecida aos participantes deve representar cada uma das dimensões teóricas de interesse para o estudo (Lieberman, 1987); (2) cada situação-estímulo deve se aproximar ao máximo de uma decisão ou julgamento a ser tomado na vida real do participante (Alexander \& Becker, 1978; Barter \& Renold, 1999; Lieberman, 1987)e ser desenvolvida com base na literatura, da mais detalhada possível (Wilson \& While, 1998); (3) no caso de haver restrições para se descrever uma dada situação-estímulo, devido a sua especificidade, recomenda-se que as vinhetas sejam desenvolvidas de maneira colaborativa com especialistas nesta técnica (Lieberman, 1987; Wilson \& While, 1998); (4) é importante a realização de um pré-teste para garantir a aderência do conteúdo da vinheta com a situação estudada (Wilson \& While, 1998); (5) o contexto das vinhetas deve ser suficiente para que os participantes compreendam a situação-estímulo, no entanto deve ser vago o suficiente para que os participantes "sejam forçados" a acrescentar elementos que influenciam suas decisões (Barter \& Renold, 1999); (6) o formato das vinhetas deve ser ajustado a cada situação estudada, a despeito de as vinhetas escritas do tipo narrativo serem as mais comuns, também podem ser apresentados na forma de vídeo (Barter \& Renold, 1999); (7) em algumas circunstâncias, pode ser desejável incluir uma vinheta de controle para ver se surgem quaisquer diferenças significativas na posição dos participantes (Barter \& Renold, 1999, 2000). 


\subsubsection{Benefícios do uso de vinhetas}

Um dos principais benefícios associados ao uso de vinhetas em pesquisas de ciências sociais está relacionado ao fato de que elas podem ser vistas como uma alternativa ao nível de abstração resultante de levantamentos realizados por meio de entrevistas e questionários, tornando-os menos vagos e mais próximos de uma tomada de decisão na vida real.

Adicionalmente, o uso de vinhetas permite ao pesquisador um maior grau de uniformidade e controle da situação estímulo (Alexander \& Becker, 1978), proporcionando o uso de representações seletivas da realidade, aumentando o foco do estudo, uma vez que as informações desejadas podem ser definidas e incluídas em função de requisitos específicos da sua pesquisa (Hughes \& Huby, 2001).

Outro aspecto positivo que decorre do uso de vinhetas é que elas permitem ao pesquisador coletar dados de um número maior de participantes, o que as tornam uma solução interessante para pesquisas com recursos limitados e orçamentos restritos, principalmente quando os custos envolvidos em outras abordagens de pesquisa são consideráveis (Wilson \& While, 1998).

\section{CONTEXTO DO ESTUDO}

Esta pesquisa foi realizada no ano de 2017, em empresas do setor farmacêutico brasileiro. Este setor é especialmente atraente para estudos desta natureza devido à quantidade e variedade de iniciativas de colaboração, realizadas por meio de alianças estratégicas para o desenvolvimento, comercialização e distribuição de produtos entre empresas da cadeia farmacêutica.

Esse fato está intrinsecamente ligado às características estruturais das organizações do setor, no qual as alianças têm um papel central, devido à possibilidade de complementaridade de competências, divisão dos riscos, compartilhamento dos custos de inovação e aumento do desempenho econômico. As características estruturais, somadas às transformações do ambiente macroeconômico e institucional, bem como o aumento de complexidade das tecnologias, da necessidade de redução do ciclo de vida dos produtos e do foco de muitas empresas nas competências centrais, fazem com que as empresas do setor farmacêutico vejam nas alianças estratégicas uma fonte de vantagem competitiva e de bom desempenho econômico (Santos \& Pinho, 2012).

\section{METODOLOGIA}

Este estudo foi operacionalizado por meio de uma estratégia de investigação qualitativa do tipo básica (Godoi \& Balsini, 2006), utilizando-se a técnica de vinhetas para compreender como os participantes atribuem significados ao fenômeno estudado, por meio do estabelecimento de padrões recorrentes e temas que atravessam os dados (Barter \& Renold, 1999). Assim, buscouse explorar as percepções de quatro gestores de alianças em quatro empresas da indústria farmacêutica brasileira, conforme mostrado na Tabela 3. Os participantes foram escolhidos por 
meio de amostragem intencional e por serem elementos "típicos" e "representativos" para o estudo. O tamanho da amostra intencional foi determinado pelo critério de saturação teórica das categorias estudadas.

Tabela 3. Lista de participantes da investigação qualitativa

\begin{tabular}{|c|c|c|c|c|c|c|c|}
\hline $\begin{array}{l}\text { Partic- } \\
\text { ipante }\end{array}$ & Gênero & $\begin{array}{l}\text { Idade } \\
\text { (anos) }\end{array}$ & Escolaridade & Cargo & $\begin{array}{l}\text { Tempo na } \\
\text { Função }\end{array}$ & $\begin{array}{l}\text { Tipo de } \\
\text { Empresa }\end{array}$ & Porte \\
\hline 01 & Masculino & 50 & Pós-Graduação & $\begin{array}{l}\text { Diretor } \\
\text { de Novos } \\
\text { Negócios }\end{array}$ & 10 & Biotecnologia & Pequeno \\
\hline 02 & Masculino & 45 & Pós-Graduação & $\begin{array}{l}\text { Gerente de } \\
\text { Projetos de } \\
\text { Parcerias }\end{array}$ & 5 & $\begin{array}{l}\text { Laboratório } \\
\text { Farmacêutico }\end{array}$ & Grande \\
\hline 03 & Masculino & 47 & Superior & $\begin{array}{l}\text { Gerente } \\
\text { de Novos } \\
\text { Mercados }\end{array}$ & 17 & $\begin{array}{l}\text { Laboratório } \\
\text { Farmacêutico }\end{array}$ & Grande \\
\hline 04 & Masculino & 55 & Superior & $\begin{array}{l}\text { Gerente de } \\
\text { P\&D }\end{array}$ & 10 & $\begin{array}{l}\text { Laboratório } \\
\text { Farmacêutico }\end{array}$ & Grande \\
\hline
\end{tabular}

Fonte: elaborada pelo autor.

Na coleta de dados, após a garantia ao direito de proteção da confidencialidade dos dados e da obtenção e registro de maneira voluntária da autorização de cada participante para a realização do estudo, foram oferecidas duas vinhetas (Tabela 4) para cada participante com o objetivo de explorar as dimensões teóricas de interesse. Essas vinhetas projetam situações-estímulo que evidenciam eventos críticos e raros que representam fatores de importância para a tomada de decisão na situação estudada.

Em seguida, foram conduzidas as entrevistas com cada um dos participantes, o armazenamento de cada uma das entrevistas em mídia digital e a transcrição literal das entrevistas. 
Neste trabalho, assumiu-se a perspectiva da entrevista como um evento de intercâmbio dialógico capaz de enriquecer a prática de pesquisa e de construir novas situações de conhecimento (Godoi \& Mattos, 2006).

Tabela 4. Vinhetas e dimensões teóricas de interesse

Vinheta
Vinheta 1: Faz dois anos que o laboratório Verificação das dimensões tipo e atribuição
farmacêutico NUBLIN, por meio de uma joint do evento prejudicial à reputação em uma
venture com a empresa de biotecnologia OBERON, situação crítica de grande visibilidade.
desenvolve um medicamento biotecnológico
inovador para o controle da diabetes. Luísa é
diretora de P\&D do laboratório farmacêutico NUBLIN
e acabou de ficar sabendo que outro medicamento
desenvolvido pela empresa OBERON teve a sua
comercialização suspensa pela ANVISA, devido a
efeitos negativos para a saúde de pacientes e que
este assunto está repercutindo fortemente na mídia.
Que tipo de consequências este acontecimento
pode trazer para a parceria da NUBLIN com a
OBERON? Como Luísa deve agir? Por que?
Vinheta2: Umadas maioresparcerias emandamento Verificação das dimensões tipo e atribuição
do laboratório KELAWAN é com a empresa de do evento prejudicial à reputação em uma
biotecnologia MIDKEMIA, para transformar um situação crítica de grande visibilidade.
cosmético em um produto pós-cirúrgico baseado
em nanotecnologia. Notícias recentes de ampla
repercussão da mídia dão conta de um rumoroso
processo, envolvendo a MIDKEMIA e órgãos
ambientais do governo, devido a uma violação
ambiental da MIDKEMIA em suas instalações. Você
trabalha no laboratório KELAWAN e é o gestor
responsável por esta parceria. Em sua opinião,
que tipo de consequências este acontecimento
pode trazer para a parceria da KELAWAN com a
MIDKEMIA? Quais seriam as suas ações neste
caso? Por que?

Fonte: elaborada pelo autor.

Com relação ao processo de análise de dados, neste estudo optou-se pela utilização da abordagem de análise de categorias (Flores, 1994), que preconiza a seguinte sequência de 
atividades realizadas com apoio do aplicativo NVivo, versão 11: a) segmentação, codificação e categorização; b) elaboração do sistema de categorias; c) exame do conteúdo das categorias e; d) comparação da percepção dos participantes nas diversas categorias.

\section{ANÁLISE DAS CATEGORIAS}

Após a análise das quatro transcrições resultantes das entrevistas com os participantes, foram identificadas três (3) categorias, conforme descrito na Tabela 5.

Tabela 5. Lista de categorias

\begin{tabular}{ll}
\hline Tipo do Evento & $\begin{array}{l}\text { Inclusões alusões de que o tipo do evento prejudicial deve ser consid- } \\
\text { erado como um elemento que afeta negativamente a sua reputação } \\
\text { corporativa de um parceiro. }\end{array}$ \\
\hline $\begin{array}{l}\text { Atribuição de } \\
\text { Responsabilidade }\end{array}$ & $\begin{array}{l}\text { Inclusões alusões de que a atribuição de responsabilidade ao par- } \\
\text { ceiro sobre um evento prejudicial deve ser considerada como um el- } \\
\text { emento que afeta negativamente a sua reputação corporativa de um } \\
\text { parceiro. }\end{array}$ \\
\hline Repercussão do Evento & $\begin{array}{l}\text { Inclusões alusões de que a repercussão na imprensa de um evento } \\
\text { prejudicial deve ser considerada como um elemento que afeta nega- } \\
\text { tivamente a reputação corporativa de um parceiro. }\end{array}$ \\
\hline
\end{tabular}

Fonte: elaborada pelo autor.

Após a codificação e categorização das narrativas, procedeu-se a elaboração do sistema de categorias. Este processo consiste em examinar o conteúdo de cada categoria com a finalidade de fundir categorias afins, subdividir categorias excessivamente amplas e renomear categorias, buscando termos que as definam melhor. Ainda, naquele momento, buscou-se conectar as categorias entre si, de acordo com a sua afinidade temática, diferenciando-as em metacategorias (Flores, 1994). Tendo em mente essas assertivas, elaborou-se para este estudo um sistema de categorias, conforme pode ser visualizado na Figura 1. 
Figura 1. Sistema de categorias.

Fonte: elaborada pelo autor

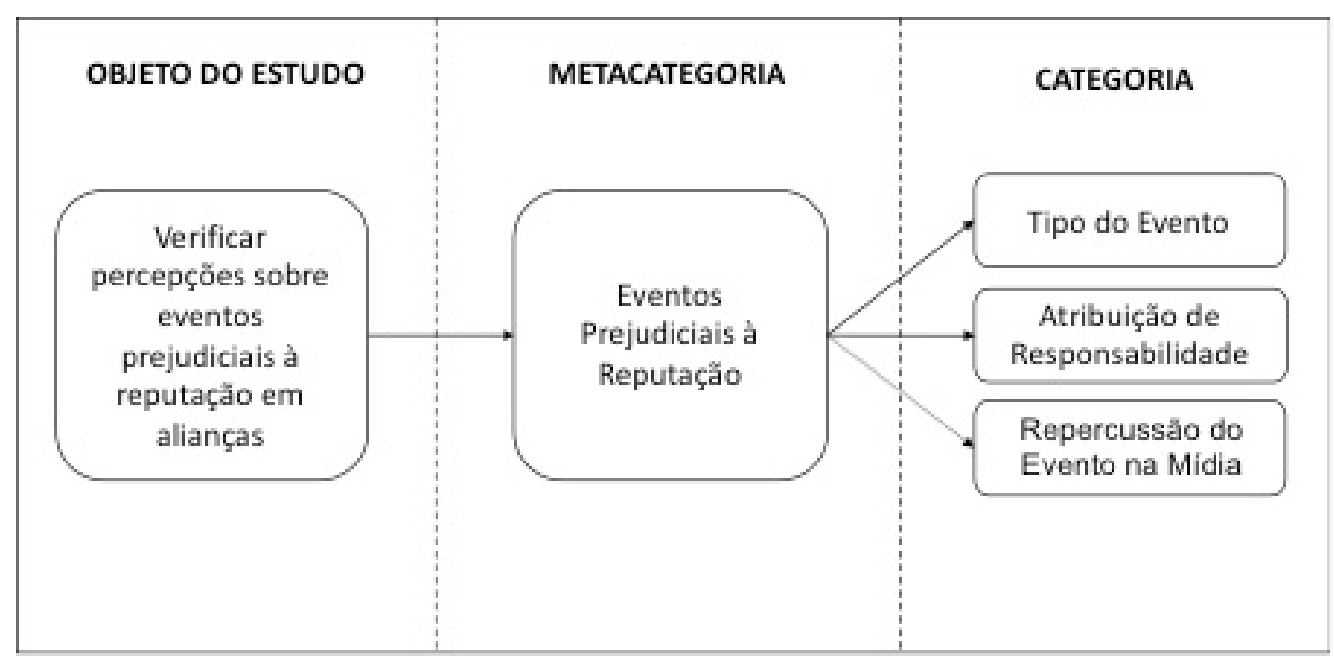

O sistema de categorias mostra uma metacategoria de eventos prejudiciais à reputação, que se subdivide em três categorias: a primeira, designada "Tipo do Evento", contendo alusões ao tipo do evento prejudicial, que deve ser considerado como um elemento que afeta negativamente a reputação corporativa de um parceiro. A segunda categoria, designada "Atribuição de Responsabilidade", contém alusões de que a atribuição de responsabilidade ao parceiro sobre um evento prejudicial deve ser considerada como um elemento que afeta negativamente a reputação corporativa de um parceiro. A terceira categoria, designada "Repercussão do Evento", contém alusões sobre a repercussão na imprensa de um evento prejudicial, que deve ser considerada como um elemento que afeta negativamente a reputação corporativa de um parceiro. Conforme descrito na Tabela 6, com o apoio do aplicativo de computador NVivo, foram examinadas em detalhes todas as referências relacionadas a cada uma das categorias que compõem a metacategoria de eventos prejudiciais à reputação. 
Tabela 6: Metacategoria eventos prejudiciais à reputação corporativa

\begin{tabular}{|c|l|r|c|}
\hline Código & \multicolumn{1}{|c|}{ Descrição da Categoria } & $\begin{array}{c}\text { Unidades } \\
\text { (Referências } \\
\text { encontradas nas } \\
\text { transcrições) }\end{array}$ & $\begin{array}{c}\text { Quantidade } \\
\text { de } \\
\text { Ocorrências }\end{array}$ \\
\hline Tipo do Evento & $\begin{array}{l}\text { Inclui alusões que indicam o tipo do evento } \\
\text { prejudicial como um elemento que afeta } \\
\text { negativamente a reputação corporativa de } \\
\text { um parceiro. }\end{array}$ & $\begin{array}{r}\text { T2R1, T2R2, T2R3, } \\
\text { T3R1, T3R2, T3R3, } \\
\text { T3R4 }\end{array}$ & 6 \\
\hline Atribuição de & $\begin{array}{l}\text { Inclui alusões que indicam a atribuição de } \\
\text { responsabilidade do parceiro com relação } \\
\text { a um evento prejudicial como um elemento } \\
\text { que afeta negativamente a reputação cor- } \\
\text { porativa de um parceiro. }\end{array}$ & $\begin{array}{l}\text { T3R1, T3R2, T4R1, } \\
\text { T4R2, T4R3, T4R4 }\end{array}$ & 6 \\
\hline $\begin{array}{l}\text { Repercussão do } \\
\text { Evento na Mídia }\end{array}$ & $\begin{array}{l}\text { Inclui alusões que indicam a repercussão } \\
\text { na imprensa sobre um evento prejudicial } \\
\text { como um elemento que afeta negativa- } \\
\text { mente a reputação corporativa de um par- } \\
\text { ceiro. }\end{array}$ & $\begin{array}{l}\text { T1R1, T1R2, T1R3, } \\
\text { T2R1, T2R2, T2R3, } \\
\text { T3R2, T4R1, } \\
\text { T4R2 }\end{array}$ & 10 \\
\hline \multicolumn{1}{|c|}{ Total } & $\mathbf{2 2}$ \\
\hline
\end{tabular}

Fonte: elaborada pelo autor.

A metacategoria dos eventos prejudiciais à reputação corporativa é composta por categorias que representam fatores que são determinantes para a percepção de que estes eventos afetam negativamente a reputação de um parceiro de negócios. Ficou claro, por meio desta investigação, que a categoria tipo do evento prejudicial à reputação corporativa é um fator que deve ser considerado pelos tomadores de decisão em alianças estratégicas. A análise dos dados indica que determinados tipos de eventos prejudiciais são considerados mais danosos à reputação do que outros, o que pode levar a não concretização da parceria, como se identifica na seguinte fala de um participante:

Por exemplo, se a empresa está envolvida em um escândalo ambiental, trabalhista. É lógico você não vai contratar... é claro que acho que não dá para ignorar, mas tem muito daquilo que eu falei antes, pois mesmo em empresas extremamente competentes já ocorreram casos em que elas se expuseram demais.

A fala do participante exprime, ainda, que eventos prejudiciais à reputação corporativa geram uma exposição indesejada dos parceiros de negócios, mesmo nos casos em que estes parceiros 
apresentam uma reputação de competência o que, por consequência, anula os possíveis benefícios que seriam obtidos com a aliança.

Na visão dos participantes, a atribuição clara de responsabilidade ao parceiro, aliada à repercussão de um evento crítico, também parece ser um fator que enfatiza os efeitos de eventos prejudiciais à reputação, como fica evidenciado na seguinte afirmação de um dos respondentes:

Tem empresa que, por mais que tente assegurar a questão da segurança, ela pode ser responsável por um vazamento -como no caso lá de Mariana - ali foi uma imprudência mesmo, pelo que se noticia foi uma imprudência - me parece que ela não está tratando de forma adequada o pós-acidente né? Então em um caso como aquele, parceria encerrada com certeza.

A fala do participante denota que a clara atribuição a um parceiro de negócios de um evento prejudicial à reputação tem influência significativa na sua percepção sobre como o evento afeta a aliança.

Outro aspecto evidenciado pela narrativa dos participantes foi que a categoria repercussão de um evento prejudicial à reputação na imprensa, por exemplo, potencializa o efeito negativo dos eventos prejudiciais sobre a reputação corporativa de um parceiro na aliança. Isto ficou evidenciado na seguinte afirmação de um participante:

O problema maior é o da publicidade que, realmente, eu acho que você disse aqui <na vinheta> teve uma repercussão forte na mídia - aí eu acho que é muito, a gestão de crise. É...., vamos lá, se a situação for extremamente grave, houve mortes ou alguma coisa assim, a empresa está sendo batida de tudo quanto é jeito, o negócio na imprensa porque isso também pode ocorrer. Existe uma ligação pública entre o que a empresa está desenvolvendo com você e a sua própria empresa - eu acho que a medida mais sensata é se afastar.

Essa fala indica claramente o peso que a repercussão de um evento prejudicial tem na tomada de decisão de um gestor de alianças, principalmente devido à possibilidade de uma indesejada associação de imagem com o parceiro que é foco do episódio negativo

\section{DISCUSSÃO DOS RESULTADOS}

A etapa qualitativa da investigação foi bem-sucedida ao explorar a percepção de gestores de alianças estratégicas sobre como eventos prejudiciais à reputação de parceiros de negócios afetam estas parcerias. Nesse sentido, foi necessário avaliar qualitativamente as categorias tipo do evento, atribuição de responsabilidade e repercussão do evento, conforme demonstrado na Tabela 7. 
Tabela 7: Síntese dos resultados da investigação qualitativa

\begin{tabular}{|c|c|c|c|}
\hline \multicolumn{4}{|c|}{ Resultados da Investigação Qualitativa } \\
\hline Metacategoria & Categoria & $\begin{array}{l}\text { Quantidade de } \\
\text { Referências }\end{array}$ & $\begin{array}{c}\text { É suportado pelo conceito } \\
\text { teórico }\end{array}$ \\
\hline \multirow{3}{*}{ 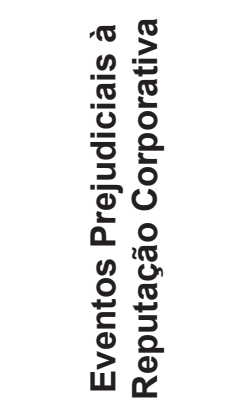 } & Tipo do Evento & 6 & SIM \\
\hline & Atribuição de Responsabilidade & 6 & SIM \\
\hline & Repercussão do Evento na Mídia & 10 & Não \\
\hline
\end{tabular}

Fonte: elaborada pelo autor.

Ao examinar a metacategoria eventos prejudiciais à reputação corporativa, os resultados evidenciam que estes eventos podem comprometer a reputação corporativa de uma organização, influenciando negativamente as percepções dos gestores de alianças estratégicas sobre a viabilidade destas parcerias. Isto confirma a ideia encontrada na teoria de que eventos prejudiciais à reputação tendem a prejudicar as percepções sobre as perspectivas de futuro de uma parceria ao criar maior incerteza ambiental. A razão para isso é que, muitas vezes, esses eventos afetam de forma negativa a credibilidade das organizações. Este fato confirma a ideia de que eventos prejudiciais à reputação acabam por resultar em erosão da confiança percebida, acarretando a perda da confiabilidade nas empresas (Gertsen et al., 2006). Entretanto, para compreender de maneira mais aprofundada esta questão é necessário examinar mais de perto as categorias que compõem a metacategoria eventos prejudiciais à reputação corporativa. Ao fazermos isso, percebemos que estas têm papel determinante neste processo.

Ao verificar os resultados da categoria tipo do evento prejudicial à reputação, percebe-se que os gestores de alianças estratégicas podem reagir de forma diferente em função da tipificação de um evento prejudicial. As narrativas dos participantes indicam que determinados tipos de eventos prejudiciais à reputação são potencialmente mais nocivos na medida em que expõem de maneira indesejada os parceiros de negócios. Esta constatação denota que estes eventos críticos têm o potencial de afetar de forma negativa as percepções das partes interessadas, dado que acabam por gerar uma visibilidade externa a respeito de falhas que foram cometidas (Rhee \& Valdez, 2009). 
Mais um aspecto a ser considerado sobre como sobre como eventos prejudiciais afetam uma aliança estratégica diz respeito à categoria sobre a atribuição de responsabilidade a um parceiro de negócios. Os resultados desta pesquisa indicam que eventos prejudiciais em que é possível atribuir de maneira clara a responsabilidade a um parceiro têm influência decisiva sobre percepção dos gestores de alianças estratégicas. É interessante denotar que isto confirma a ideia de que quando um evento prejudicial à reputação pode ser claramente imputado como culpa de uma empresa, esta atribuição potencializa a percepção de que tal empresa deve ser a responsável pelo evento, o que acaba por resultar em maiores danos à reputação da organização (Moowen Rhee \& Kim, 2012).

Por fim, a repercussão do evento prejudicial na mídia é um tema recorrente nas narrativas dos entrevistados. Os resultados sugerem que um evento prejudicial que tem repercussão na imprensa potencializa a percepção dos efeitos negativos deste evento para a aliança estratégica. De maneira geral, as falas dos participantes demonstram uma preocupação com o fato de estes terem uma associação pública com parceiros que estão envolvidos em problemas que estão sob os holofotes da imprensa e, por consequência, da opinião pública. Apesar de estudos sobre eventos prejudiciais à reputação mostrarem que uma crise tem o potencial de ameaçar a reputação de uma organização porque dá às pessoas motivos para pensarem mal da organização (Coombs \& Holladay, 2002; Timothy \& Coombs, 2007), o fato é que esta ameaça é muito amplificada quando estes eventos são repercutidos nos meios de comunicação, em especial na imprensa.

\section{CONCLUSÃO}

Este artigo soma-se ao esforço teórico de aprofundar a compreensão sobre como eventos prejudiciais à reputação afetam a percepção das partes interessadas de uma empresa e sobre as percepções dos gestores de alianças estratégicas, que interpretam eventos que impactam negativamente esta reputação. Ademais, é necessário observar a contribuição teórica deste estudo para a compreensão do conjunto de percepções dos gestores de alianças estratégicas envolvidas nos processos de interpretação sobre como eventos de natureza crítica podem afetar a manutenção da reputação corporativa nestas parcerias.

Sem a intenção de esgotar o tema, este estudo possibilitou um avanço no entendimento de como as percepções subjacentes à reputação corporativa permitem, no caso de eventos críticos, destruir o valor da reputação para as organizações, na medida em que influenciam, de maneira negativa, a credibilidade e consequente desempenho da aliança estratégica. Neste sentido, este estudo contribui com a teoria, adicionando o conhecimento sobre o papel da repercussão de eventos nos meios de comunicação como uma dimensão importante dos eventos prejudiciais à reputação. $A$ repercussão na mídia amplifica, negativamente, a percepção de eventos prejudiciais ocorridos com parceiros de negócio, afetando de maneira determinante o padrão de decisão dos gestores destas alianças.

Também cabe ressaltar as implicações deste estudo para a metodologia, ao utilizarem-se vinhetas na pesquisa qualitativa com a finalidade de explorar o conhecimento socialmente adquirido e 
práticas interpretativas dos participantes. Em especial, ressalta-se a adequação do uso desta técnica em estudos que tenham a necessidade de elucidar os processos de significados e de interpretações realizados pelos participantes, ao se depararem com situações que exigem uma tomada de decisão ou julgamento.

Neste sentido, este estudo empírico foi bem-sucedido e avança o conhecimento adquirido em estudos prévios (Autor A \& Autor B, 2018), ao demonstrar que as vinhetas consistem em uma técnica de coleta de dados ideal para ser utilizada em pesquisas qualitativas que queiram provocar o posicionamento dos participantes a partir de determinas situações-estímulo. Ao sugerir aos participantes que se posicionem perante as vinhetas que lhes foram oferecidas, assume-se que irão reinserir as narrativas descontextualizadas em seu próprio contexto, de maneira que isso possibilita aos pesquisadores um diálogo mais próximo da ideia de pesquisa qualitativa, em que pesquisador e sujeito constroem mutuamente o material de pesquisa.

Contudo, é importante levar-se em conta as possíveis limitações do uso de vinhetas em uma investigação qualitativa. Alguns pesquisadores argumentam que as vinhetas apresentam certa artificialidade, quando comparadas com outras abordagens, como a observação, por exemplo (Wilson \& While, 1998). Assim, a relação entre a vinheta e a "realidade social", entre a crença (aquilo que o participante diz que "deve" fazer) e a ação (o que o participante "realmente" faz) também é apontada como uma limitação teórica e metodológica para o uso desta técnica, o que faz com que alguns pesquisadores tragam alertas para o uso das vinhetas como um método autônomo de pesquisa (Faia, 1980).

Entretanto, cabe argumentar que não são o resultado ou a ação os interesses de pesquisa, mas sim o processo de significados e interpretações utilizados para alcançar o resultado. Esta deve ser a preocupação central dos cientistas sociais.

\section{REFERÊNCIAS}

Alexander, C., \& Becker, H. (1978). The use of vignettes in survey research. Public Opinion Quarterly, 42, 93.

Ashta, A., Stokes, P., \& Hughes, P. (2018). Change management in Indo-Japanese cross-cultural collaborative contexts: Parallels between traditional Indian philosophy and contemporary Japanese management. Journal of Organizational Change Management, 31, 230-247.

Barney, J. (1991). Firm Resources and Sustained Competitive. Journal of Management, 17, 99-120.

Barter, C., \& Renold, E. (1999). The Use of Vignettes in Qualitative Research. Social Research Update, $1-7$.

Barter, C., \& Renold, E. (2000). "I wanna tell you a story": Exploring the application of vignettes in qualitative research with children and young people. International Journal of Social Research Methodology, 3, 307-323. 
Berg Johansen, C., \& De Cock, C. (2018). Ideologies of time: How elite corporate actors engage the future. Organization, 25, 186-204.

Coombs, W. T. (2007). Protecting organization reputations during a crisis: The development and application of situational crisis communication theory. Corporate Reputation Review, 10, 163-176.

Coombs, W. T., \& Holladay, S. J. (2002). Helping Crisis Managers Protect Reputational Assets: Initial Tests of the Situational Crisis Communication Theory. Management Communication Quarterly, 16, 165-186.

Crafter, S., De Abreu, G., Cline, T., \& O'Dell, L. (2010). Using the vignette methodology as a tool for exploring cultural identity positions. European Association for Research on Learning and Instruction (EARLI) Special Interest Group 21: Learning and Teaching in Culturally Diverse Settings: Moving through Cultures of Learning, 2-3.

Dollinger, M. J., Golden, P. A., \& Saxton, T. (1997). The Effect of Reputation on the Decision to Joint Venture. Strategic Management Journal, 18, 127-140.

Eskelinen, L., \& Caswell, D. (2006). Comparison of Social Work Practice in Teams Using a Video Vignette Technique in a Multi-method Design. Qualitative Social Work, 5, 489-503.

Feldman, P. M., Bahamonde, R. A., \& Bellido, I. V. (2014). A new approach for measuring corporate reputation. RAE-Revista de Administração de Empresas, 54, 53-67.

Finch, J., \& Mason, J. (1991). Obligations of kinship in contemporary Britain: is there normative agreement? The British Journal of Sociology, 42, 345-367.

Flores, J. G. (1994). Análisis datos cualitativos: Aplicaciones a investigación educativa. Barcelona: Latorre Literaria.

Fombrun, C. J. (1996). Reputation: Realizing Value from the Corporate Image. The Service Industries Journal, 10, 99-101.

Fombrun, C. J., Gardberg, N. A., \& Sever, J. W. (2000). The Reputation QuotientSM : A Multi-Stakeholder Measure of Corporate Reputation. The Journal of Brand Management, 7, 241-255.

Fombrun, C. J., \& Shanley, M. (1990). What'S in a Name? Reputation Building and Corporate Strategy. Academy of Management Journal, 33, 233-258.

Gatzert, N. (2015). The impact of corporate reputation and reputation damaging events on financial performance: Empirical evidence from the literature. European Management Journal, 33, 485-499.

Gatzert, N., Schmit, J. T., \& Kolb, A. (2016). Assessing the Risks of Insuring Reputation Risk. Journal of Risk and Insurance, 83, 641-679.

Gertsen, F. H. M., van Riel, C. B. M., \& Berens, G. (2006). Avoiding Reputation Damage in Financial Restatements. Long Range Planning, 39, 429-456.

Godoi, C. K., \& Balsini, C. P. V. (2006). A visão qualitativa nos estudos organizacionais: elementos metodológico-epistemológicos. In Pesquisa qualitativa em estudos organizacionais: paradigmas, estratégias e métodos. (pp. 93-96). São Paulo: Saraiva. 
Gourlay, A., Mshana, G., Birdthistle, I., Bulugu, G., Zaba, B., \& Urassa, M. (2014). Using vignettes in qualitative research to explore barriers and facilitating factors to the uptake of prevention of mother-to-child transmission services in rural Tanzania: a critical analysis. BMC Medical Research Methodology, 14, 21.

Hirsch, B., \& Meyer, M. (2010). Integrating soft factors into the assessment of cooperative relationships between firms: Accounting for reputation and ethical values. Business Ethics, 19, 81-94.

Autor A, \& Autor B (2018). Utilização de Vinhetas para a Coleta de Dados: Aplicação em um Estudo Exploratório sobre Eventos Prejudiciais à Reputação. Atas CIAIQ2018, 7o. Congresso IberoAmericano de Investigação Qualitativa, 3, 47-56.

Hughes, R. (1998). Considering the Vignette Technique and its Application to a Study of Drug injecting and HIV Risk and Safer Behaviour. Sociology of Health \& IIIness, 20, 381-400.

Hughes, R., \& Huby, M. (2001). The application of vignettes in social and nursing research. Journal of Advanced Nursing, 37, 382-386.

Hutt, R. W. (2016). Reputation on the line: the Starbucks cases. Journal of Business Strategy, 37, 19-26.

Jenkins, N. (2006). Developing interactive vignettes in a study of young people's injury-risking behavior. Qualitative Researcher, 7-8.

Jenkins, N., Bloor, M. J., Fischer, J., Berney, L., \& Neale, J. (2010). Putting it in context: the use of vignettes in qualitative interviewing. Qualitative Research, 10, 175-198.

Lieberman, A. (1987). Documenting Professional Practice: The Vignette as a Qualitative Tool. In Annual Meeting of the American Educational Research Association (pp. 1-14). Washington: Annual Meeting of the American Educational Research Association.

Mariano, S. (2018). Initiating, challenging and improving knowledge in organizational contexts. Management Research Review, 41, 278-295.

Miles, M. B. M., \& Huberman, A. M. (1994). Qualitative Data Analysis: A Methods Sourcebook. (Thousand Oaks, Ed.). CA: Sage.

Renold, E. (2002). Using Vignettes in Qualitative Researsch. Building Research Capacity, 3-5.

Reputation Institute. (2016). Reputation Leaders Study 2016: How Global Executives Grapple with Today's Reputation Risks and Capitalize on Opportunities.

Rhee, M., \& Kim, T. (2012). After the collapse: A behavioral theory of reputation repair. In The Oxford Handbook of Corporate Reputation (pp. 446-465). Oxford, UK: Oxford University Press.

Rhee, M., \& Valdez, M. E. (2009). Contextual Factors Surrounding Reputation Damage With Potential Implications for Reputation Repair. The Academy of Management Review, 34, 146-168.

Roberts, P. W., \& Dowling, G. R. (2002). Corporate reputation and sustained superior financial performance. Strategic Management Journal, 23, 1077-1093.

Santos, M. C. G., \& Pinho, M. (2012). Estratégias tecnológicas em transformação: um estudo da indústria farmacêutica brasileira. Gestão \& Produção, 19, 405-418. 
Spalding, N. J., \& Phillips, T. (2007). Exploring the use of vignettes: from validity to trustworthiness. Qualitative Health Research, 17, 954-962.

Sydow, J. (1998). Understanding the Constitution of Interorganizational Trust. In Trust within and between organizations: Conceptual issues and empirical applications. (pp. 12-14). Oxford, UK: Oxford University Press.

Tettegah, S., Bailey, B. P., \& Taylor, K. (2007). Clover: Narratives and Simulations in Virtual Environments. The Journal of Negro Education, 76, 43-56.

Wagner, S. M., Coley, L. S., \& Lindemann, E. (2011). Effects of suppliers' reputation on the future of buyersupplier relationships: The mediating roles of outcome fairness and trust. Journal of Supply Chain Management, 47, 29-48.

Wilson, J., \& While, A. E. (1998). Methodological issues surrounding the use of vignettes in qualitative research. Journal of Interprofessional Care.

Wright, J. M., Heathcote, K., \& Wibberley, C. (2014). Fact or fiction: Exploring the use of real stories in place of vignettes in interviews with informal carers. Nurse Researcher, 21, 39-43. 